\title{
Chiral Differentiation of Some Cyclopentane and Cyclohexane $\beta$-Amino Acid Enantiomers Through Ion/Molecule Reactions
}

\author{
Anna R. M. Hyyryläinen, ${ }^{a}$ Jaana M. H. Pakarinen, ${ }^{\text {a Enikő Forró, }}{ }^{\text {b }}$ \\ Ferenc Fülöp, ${ }^{b}$ and Pirjo Vainiotalo ${ }^{a}$ \\ a Department of Chemistry, University of Joensuu, Joensuu, Finland \\ ${ }^{b}$ Institute of Pharmaceutical Chemistry, University of Szeged, Szeged, Hungary
}

Chiral differentiation of four enantiomeric pairs of $\beta$-amino acids, cis-(1R,2S)-, cis-(1S,2R)-, trans-(1R,2R)-, and trans-(1S,2S)-2-aminocyclopentanecarboxylic acids (cyclopentane $\beta$-amino acids), and cis-(1R,2S)-, cis-(1S,2R)-, trans-(1R,2R)-, and trans-(1S,2S)-2-aminocyclohexanecarboxylic acids (cyclohexane $\beta$-amino acids) was performed successfully by using host-guest complexes and ion/molecule reactions. The experiments were conducted by using electrospray ionization Fourier transform ion cyclotron resonance mass spectrometry. The effect of a chiral host molecule was tested by using three different host compounds; (+)-(18-Crown-6)-2,3,11,12-tetracarboxylic acid, $(-)-(18$-Crown-6)-2,3,11,12-tetracarboxylic acid, and $\beta$-cyclodextrin. This is the first time that small enantiomeric pairs with two chiral centers have been differentiated using ion/molecule reactions and host-guest complexes. (J Am Soc Mass Spectrom 2009, 20, 1235-1241) () 2009 American Society for Mass Spectrometry

$\mathrm{I}$

$\mathrm{n}$ the field of host-guest chemistry, especially crown ethers and cyclodextrins (CD) have been used as host compounds when studying noncovalent interactions and inclusion complexes [1,2]. Crown ethers are synthetic macrocyclic polyethers that are mentioned as good host compounds for both cations and neutral molecules [2]. The structure of crown ethers allows the coordination of a cation via oxygen atoms located at the interior of the ring, whereas the exterior of the ring is hydrophobic. Another feasible group of host compounds, cyclodextrins, belong to a family of cyclic oligosaccharides composed of six or more D-(+)glucose units with $\mathrm{C} 1$ chair conformation, where the units are linked by $\alpha-1,4$-glycoside bonds [3-5]. The cavity of the cyclodextrin is relatively hydrophobic, while the rim is hydrophilic. Thus, cyclodextrins readily form inclusion complexes with various guest compounds, which make them highly suitable for acting as a host compound [1,5].

One important application for crown ethers and cyclodextrins has been the differentiation of enantiomers. Differentiation of enantiomers has been widely evaluated by using different chromatographic methods, especially high-performance liquid chromatography (HPLC) and capillary electrophoresis (CE) [6-10]. Chiral crown ether derivatives have been used in liquid chromatography as stationary phases [11, 12]. In addition, chiral crown ether derivatives have been used as a

Address reprint requests to Professor P. Vainiotalo, University of Joensuu, Department of Chemistry, P.O. Box 111, FI-80101 Joensuu, Finland. E-mail: pirjo.vainiotalo@joensuu.fi selector for the separation of chiral amino acids in capillary electrophoresis $[13,14]$. It has been shown that the crown ether derivative, $(+)-(18-$ Crown- 6$)-2,3,11,12-$ tetracarboxylic acid, is a very powerful selector, especially for the differentiation of compounds that have a primary amino group [14]. Cyclodextrins have also been used widely in different chromatographic methods for enantiomeric separation [15-17].

Although chromatographic methods are common in the field of enantiomeric separation, the procedure can often be very time-consuming and usually requires a lot of sample. Mass spectrometry, instead, can be very fast, sensitive, and sample friendly. Different mass spectrometric methods, especially the kinetic method presented by Cooks et al., have gained much interest in the field of enantiomer differentiation [18, 19]. Methods that utilize host-guest complexes and ion/molecule reactions have been used as well [20-28]. In the field of host-guest complexes and ion/molecule reactions, especially Dearden et al. have utilized chiral crown ether derivatives in chiral recognition of enantiomeric amines [29-31]. Moreover, Lebrilla et al. have utilized cyclodextrins as host compounds when differentiating enantiomeric $\alpha$-amino acids, pharmaceutical compounds, and peptides [32-35].

In this study, the enantiomeric differentiation of cyclic $\beta$-amino acids (1-8) was performed by using ion/molecule reactions and host-guest complexes. Four enantiomeric pairs of $\beta$-amino acids were studied: cis-(1R,2S)-, cis-(1S,2R)-, trans-(1R,2R)-, and trans$(1 S, 2 S)$-2-aminocyclopentanecarboxylic acids, and cis$(1 R, 2 S)-$, cis-(1S,2R)-, trans-(1R,2R)-, and trans-(1S,2S)-2- 


\section{cis-Isomers}<smiles>N[C@@H]1CCC[C]1C(=O)O</smiles>

$1(1 R, 2 S)$<smiles>N[C@@H]1CCC[C@H]1C(=O)O</smiles>

$2(1 S, 2 R)$

\section{trans-Isomers}<smiles>N[C@@H]1CCCC1C(=O)O</smiles><smiles>NC1CCC[C@H]1C(=O)O</smiles>

$3(1 R, 2 R)$

$4(1 S, 2 S)$

\section{cis-Isomers}<smiles>N[C@H]1CCCC[C]1C(=O)O</smiles><smiles>N[C@@H]1CCCC[C@H]1C(=O)O</smiles>

$5(1 R, 2 S)$ $6(1 S, 2 R)$

trans-Isomers<smiles>N[C@H]1CCCC[C@H]1C(=O)O</smiles>

$7(1 R, 2 R)$<smiles>NC1CCCC[C@H]1C(=O)O</smiles>

$\mathbf{8}(1 S, 2 S)$

Figure 1. $\beta$-Amino acids studied (1-8).

aminocyclohexanecarboxylic acids (Figure 1). Several chiral host compounds were tested to evaluate the enantioselectivity. The host compounds used in this study were $(+)-(18$-Crown-6)-2,3,11,12-tetracarboxylic acid $[(+)$-Crown derivative], (-)-(18-Crown-6)-2,3, 11,12-tetracarboxylic acid [(-)-Crown derivative], and $\beta$-cyclodextrin (Figure 2). In ion/molecule reactions, mainly $n$-propylamine $\left(n-\mathrm{PrNH}_{2}\right)$ was used as a neutral reagent, but triethylamine $\left(\mathrm{Et}_{3} \mathrm{~N}\right)$ was used with cyclodextrins.

Moreover, different cyclodextrins were tested, including $\alpha$-, as well as heptakis(2,3,6-tri-O-methyl)- $\beta$ cyclodextrin and maltoheptaose, a linear analog to $\beta$-cyclodextrin. In the case of these host compounds, however, the reaction times were too long or the separation of enantiomers was not successful; they are only mentioned here as a curiosity. Thus, the emphasis is on the results obtained with crown ether derivatives and $\beta$-cyclodextrin.

The chiral $\beta$-amino acids studied (Figure 1 ) have attracted much interest due to their ability to work as stabilization agents in peptides, as modifying agents of peptides with biological activity, and as starting

substances for different heterocycles [36, 37], just to mention a few applications. Especially $(1 R, 2 S)-2-$ aminocyclopentanecarboxylic acid (cispentacin), which was isolated from Bacillus cereus and Streptomyces setonii, acts as an antifungal antibiotic [37].

In our earlier study, host-guest complexes and ion/ molecule reactions were performed for the differentiation of diastereomeric $\beta$-amino acids, including cis- and trans-isomers of cyclopentane and cyclohexane $\beta$-amino acids [38]. So, in view of achieving chiral differentiation of the studied $\beta$-amino acids (1-8), the same method was applied.

\section{Experimental}

Cyclopentane and cyclohexane $\beta$-amino acids (Figure 1) were synthesized in the University of Szeged, Hungary $[9,36]$. Crown ethers, (+)-(18-Crown-6)-2,3, 11,12-tetracarboxylic acid, and (-)-(18-Crown-6)2,3,11,12-tetracarboxylic acid were obtained from Aldrich Chemical Co. (Milwaukee, WI). $\alpha$-Cyclodextrin ( $\geq 98 \%$ ), $\beta$-cyclodextrin ( $\geq 99 \%)$, heptakis $(2,3,6$-tri-Omethyl)- $\beta$-cyclodextrin ( $\geq 98 \%$ ), and maltoheptaose $(\geq 90 \%)$ were obtained from Sigma-Aldrich Co. (St. Louis, MO). The $n$-propylamine, and triethylamine

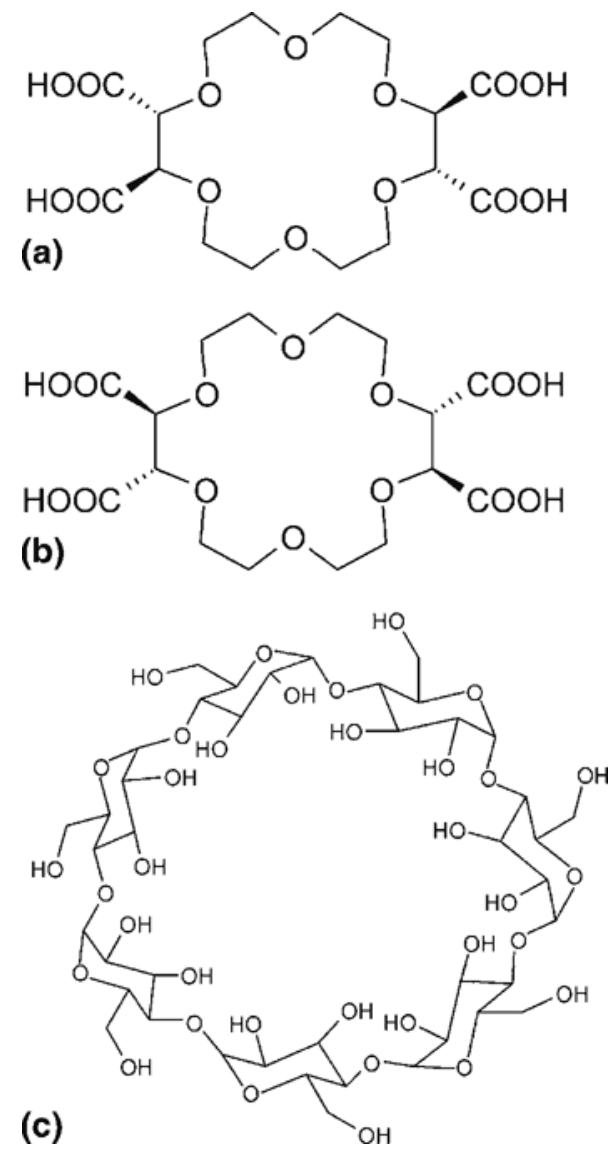

Figure 2. Host compounds used in this study. (a) (+)-(18-crown6)-2,3,11,12-tetracarboxylic acid, (b) (-)-(18-crown-6)-2,3,11,12tetracarboxylic acid, and (c) $\beta$-cyclodextrin. 
used as neutral reagents in ion/molecule reactions were also obtained from Sigma-Aldrich Co.

The host and guests were dissolved in methanol $(\mathrm{MeOH})$ at a concentration of $1 \mathrm{mM}$. The complexes were prepared by mixing the host and guest mainly at a ratio of 1:1 (in some cases 1:3), the final concentrations of host and guest in the methanol solution being $1.0 \times$ $10^{-5} \mathrm{M}$. All the mass spectrometry experiments were performed with a 4.7 T Bruker APEX-Qe hybrid Fourier transform ion cyclotron resonance mass spectrometer (Bruker Daltonics, Billerica, MA) with a mass-selective quadrupole interface. Ions were produced in an external Apollo II electrospray ionization (ESI) source. The base pressure of $1.0 \times 10^{-10}$ Torr was maintained by rotary vacuum and turbomolecular pumps supplied by Edwards (Edwards High Vacuum Int., Crawley, UK) and Pfeiffer (Pfeiffer Vacuum Technology AG, Asslar, Germany). The sample was introduced to the mass spectrometer via a $70^{\circ}$ off-axis sprayer at a rate of 90 $\mu \mathrm{L} / \mathrm{h}$. Nitrogen gas heated at $240^{\circ} \mathrm{C}$ was used as the nebulization and counter-current drying gas. The measurements and data handling were performed with the Bruker XMASS software version 7.0.8.

In ion/molecule reactions, the neutral reagent was degassed in the vacuum manifold in at least three freeze-thaw cycles before it was leaked to the analyzer cell through a variable leak valve. The pressure of the cell was allowed to rise to $5.0 \times 10^{-8}$ Torr due to the neutral reagent, and was kept constant during the measurements. The host-guest complex ion was isolated by the CHEF procedure [39] and then allowed to undergo a guest-exchange reaction with the gaseous neutral reagent. The reaction time varied from $0.1 \mathrm{~s}$ to as much as $800 \mathrm{~s}$. The spectra usually consisted of 16 summed scans with a dataset of $256 \mathrm{k}$, but also eight, six, or four scans were carried out with longer reaction times. Identical conditions were employed for the enantiomeric pairs. The errors of the enantioselectivities are presented as a relative error of the slopes.

\section{Results and Discussion}

The study showed that differentiation of chiral $\beta$-amino acids (1-8) was obtained with host-guest complexes and ion/molecule reactions, and different chiral host compounds had some effect on the enantioselectivity values obtained. Chiral crown ether derivatives and $\beta$-cyclodextrin (Figure 2) were selected because of their good ability to form host-guest complexes, as well as to recognize and differentiate enantiomers, especially using different chromatographic and mass spectrometric methods [11-17, 28-35]. The same host compounds were used for both cyclopentane $\beta$-amino acids (1-4) and cyclohexane $\beta$-amino acids (5-8).

In general, in the gas phase the $[\text { Host: } \beta-\mathrm{AC}+\mathrm{H}]^{+}$ complex ions are assumed to react with a neutral reagent (Reagent) at different rates, forming the complex ion [Host:Reagent $+\mathrm{H}^{+}$, as can be seen in eq 1 .

$$
\begin{array}{r}
{[\text { Host: } \beta \text {-AC }+\mathrm{H}]^{+}+\text {Reagent } \rightarrow} \\
{[\text { Host.Reagent }+\mathrm{H}]^{+}+\beta-\mathrm{AC}}
\end{array}
$$

The abundances of the precursor $[\text { Host: } \beta-\mathrm{AC}+\mathrm{H}]^{+}$and product [Host:Reagent $+\mathrm{H}]^{+}$ions were measured as a function of time, and rate constants $(k)$ were obtained from the slopes of the pseudo-first-order rate plots, $\ln \mathrm{I} / \mathrm{I}_{0}$ versus $t$, where I is the intensity of the precursor complex at time $t$ and $\mathrm{I}_{0}$ is the sum of the intensities of the precursor and product complex [33]. The enantioselectivity $\left(S_{c}\right)$, in turn, is defined as the ratio of the rate constants $k_{\mathrm{RS}} / k_{\mathrm{SR}}$ for the cis-isomers, and $k_{\mathrm{RR}} / k_{\mathrm{SS}}$ for the trans-isomers.

\section{Differentiation of Enantiomeric \\ 2-Aminocyclopentanecarboxylic Acids (Cyclopentane $\beta$-Amino Acids)}

The enantioselectivities $\left(S_{c}\right)$ obtained for cyclopentane $\beta$-amino acids with different host compounds are presented in Table 1. The differentiation of enantiomers was obtained with all host compounds. A high selectivity value was achieved with the $(+)$-crown ether derivative for both cis- and trans-isomers. Figure 3 shows ESI mass spectra of ion/molecule reactions of host-guest complexes of the $(+)$-crown ether derivative and the trans-isomers $(1 R, 2 R$ and $1 S, 2 S)$ of cyclopentane $\beta$-amino acid (3 and 4$)$ with $n$-propylamine $\left(n-\mathrm{PrNH}_{2}\right)$, with three different reaction times. The host-guest complex ion with the $1 R, 2 R$ enantiomer (Figure $3 a$ ) changes the guest molecule faster than the corresponding complex ion with the $1 S, 2 S$ enantiomer (Figure $3 b$ ).

The corresponding reaction rate plots for the hostguest complexes of the $(+)$-crown ether derivative and 3 and 4 are presented in Figure 4 . The result means that the structure of the complex with the $1 R, 2 R$ enantiomer is kinetically more unstable than that of the complex with the $1 S, 2 S$ enantiomer, and thus the $1 R, 2 R$ enantiomer is more easily replaced by the neutral reagent than the $1 S, 2 S$ enantiomer. Also, in the case of the cis-isomers $(1 R, 2 S$ and $1 S, 2 R)$, the $1 R, 2 S$ enantiomer was replaced faster than the corresponding $1 S, 2 R$ enantiomer when the $(+)$-crown ether derivative was used as the host compound.

In general, selectivities obtained were moderate, yet adequate. A slightly better enantioselectivity was achieved for the trans-isomers $(1 R, 2 R$ and $1 S, 2 S)$ than for the cis-isomers $(1 R, 2 S$ and $1 S, 2 R)$. The reason for this

Table 1. Chiral selectivities $\left(\mathbf{S}_{\mathrm{c}}\right)$ for enantiomeric 2-aminocyclopentane carboxylic acids studied (1-4)

\begin{tabular}{lcc}
\hline & \multicolumn{2}{c}{ Chiral selectivity $\left(\mathrm{S}_{\mathrm{c}}\right)^{\mathrm{a}}$} \\
\cline { 2 - 3 } \multicolumn{1}{c}{ Host } & $\begin{array}{c}\text { cis-Isomers } \\
\text { trans-Isomers }\end{array}$ & $\begin{array}{c}\text { trans } /(1 S, 2 R) \\
(1 R, 2 R) /(1 S, 2 S)\end{array}$ \\
\hline \hline (+)-Crown derivative & $1.46 \pm 0.04$ & $1.69 \pm 0.04$ \\
(-)-Crown derivative & $0.86 \pm 0.04$ & $0.58 \pm 0.01$ \\
$\beta$-Cyclodextrin & $0.84 \pm 0.04$ & $1.23 \pm 0.05$ \\
\hline
\end{tabular}

aSelectivity is obtained as ratio of rate constants $\left(k_{\mathrm{RS}} / k_{\mathrm{SR}}\right.$ and $\left.k_{\mathrm{RR}} / k_{\mathrm{SS}}\right)$. 


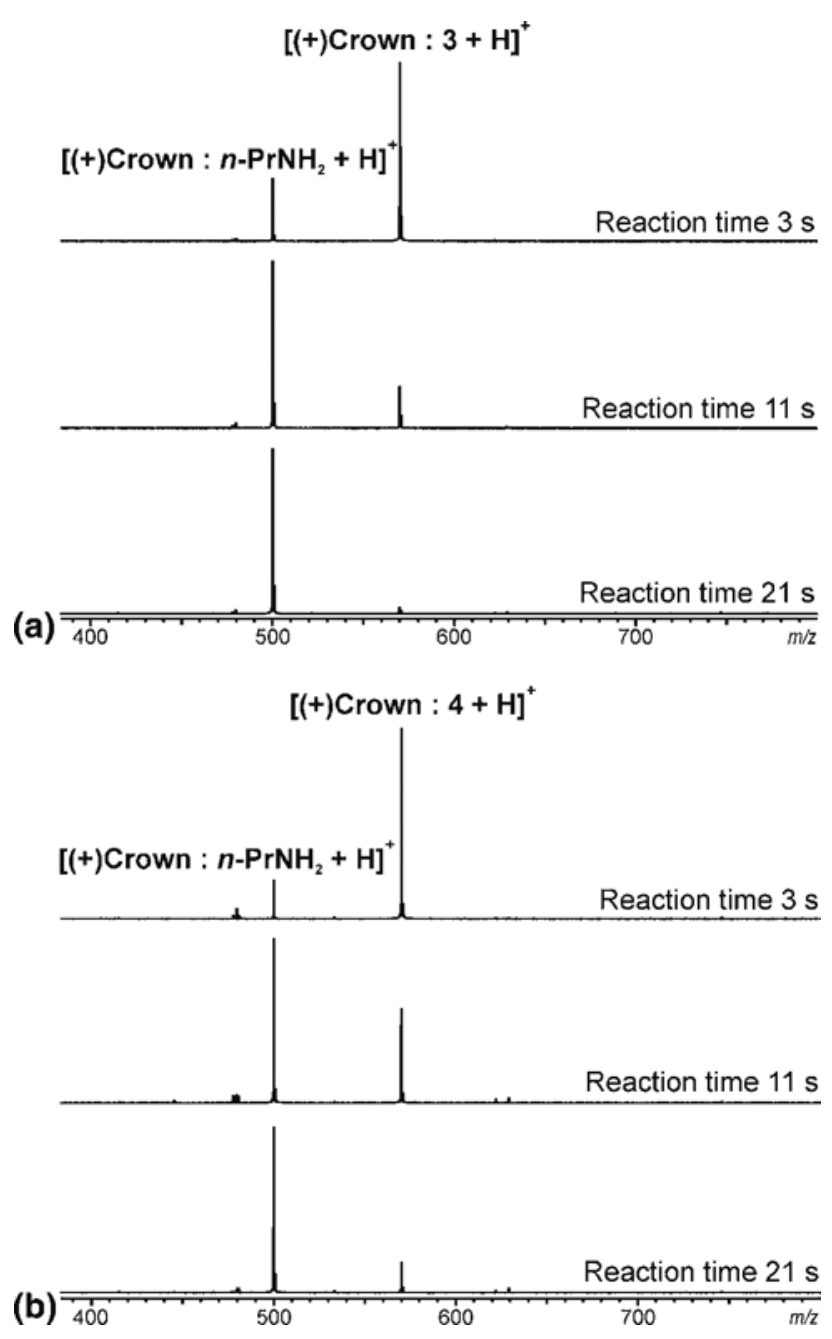

Figure 3. ESI mass spectra of ion/molecule reactions of hostcomplexes of the (+)-crown ether derivative and (a) $1 R, 2 R$ - and (b) $1 S, 2 S$-cyclopentane $\beta$-amino acids (3 and 4) with $n$ propylamine.

kind of behavior might be that with the trans-isomers, the stability differences of complexes between the enantiomers are greater, and it is thus shown in the selectivity values obtained compared with the cisisomers.

When the (-)-(18-Crown-6)-2,3,11,12-tetracarboxylic acid [(-)-Crown derivative] was used as a host molecule, the enantioselectivities obtained are similar in magnitude with results obtained with the $(+)$-crown ether derivative, but now selectivities are below one. This behavior is logical, because now the only difference is the host compound, which has a reversed optical geometry, and it is expected to bind the opposite enantiomers better than the (+)-crown ether derivative. Again, a better enantioselectivity was achieved for the trans-isomers $(1 R, 2 R$ and $1 S, 2 S)$ than for the cis-isomers $(1 R, 2 S$ and $1 S, 2 R)$.

When $\beta$-cyclodextrin ( $\beta$-CD) was used as a host molecule, the results obtained differed from previous results. First, the guest exchange reactions were con- ducted using triethylamine (proton affinity $982 \mathrm{~kJ}$ $\mathrm{mol}^{-1}$ ) as a neutral reagent. This was because $n$ propylamine (proton affinity $918 \mathrm{~kJ} \mathrm{~mol}^{-1}$ ) was not able to replace the enantiomer of interest fast enough. In fact, in some cases the reaction time was as much as $800 \mathrm{~s}$, and the guest exchange was still not adequate (the abundance of the [Host: $\beta-\mathrm{AC}+\mathrm{H}]^{+}$complex ion was still much higher than that of the [Host:Reagent $+\mathrm{H}]^{+}$ complex ion). It was observed, however, that reaction times even with triethylamine were quite long, as well (even 600 s). All these long reaction times just indicate how stable host-guest complexes are formed between $\beta$-cyclodextrin and the $\beta$-amino acids studied, compared with complexes of crown ether derivatives and the $\beta$-amino acids studied.

An interesting observation was that in almost every case in which $\beta$-cyclodextrin was used as a host compound, a trimeric complex ion was also observed in mass spectra. The trimeric complex consists of the host molecule, the enantiomer of interest, and triethylamine ([Host: $\beta$-AC:Reagent $+\mathrm{H}]^{+}$). Lebrilla et al. noticed and studied similar kinds of trimeric complexes when they separated $\alpha$-amino acid enantiomers using host-guest

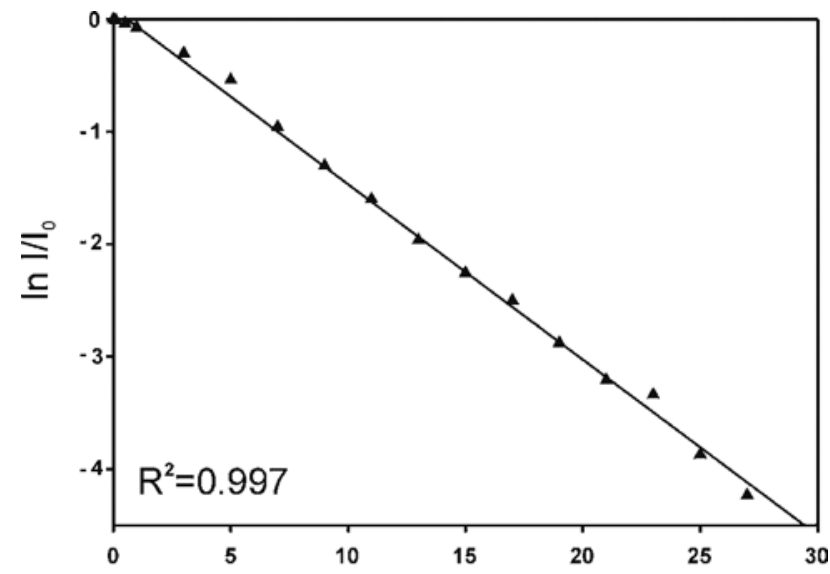

(a)

Reaction time (s)

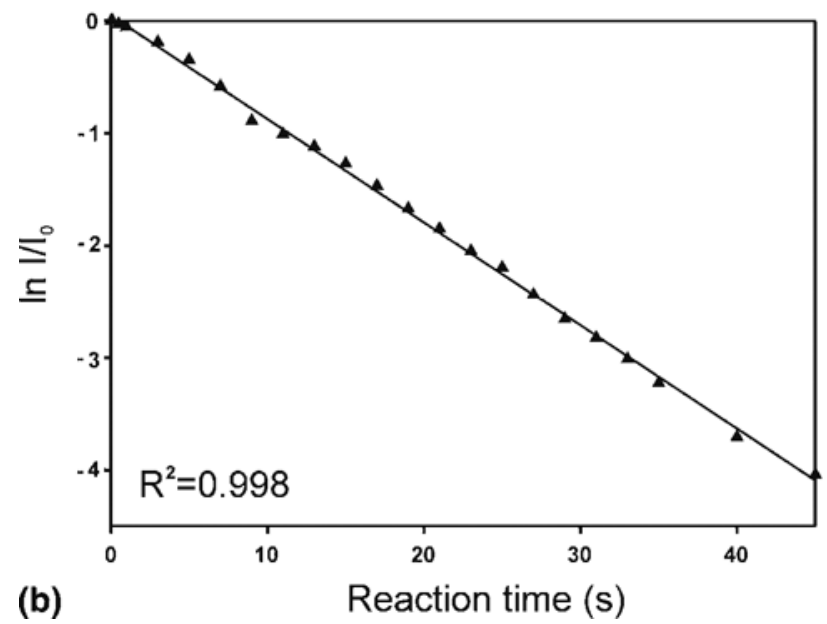

Figure 4. Rate plots for reactions between complexes of the (+)-crown ether derivative with (a) $1 R, 2 R-$ and (b) $1 S, 2 S-$ cyclopentane $\beta$-amino acids ( 3 and 4 ) and $n$-propylamine. 
complexes and ion/molecule reactions [40,41]. They assumed that these trimeric complexes have a zwitterionic nature, in which the positive charge of the protonated amine is coordinated with the carboxyl group of the amino acid. Moreover, the zwitterionic amino acids are stabilized through hydrogen bonding to the lower rim of cyclodextrin [41]. In this study, however, this phenomenon and the possible structures of trimeric complexes were not further speculated on.

Because trimeric complexes were observed, rate plots were constructed including the trimeric complex. In other words, the abundance of the trimeric complex ion was added to the sum of the intensities $\mathrm{I}_{0}$. The abundance of the trimeric complex ion, however, was minor, and thus did not affect the reaction rate plots. In addition, it was observed that the reaction times were much longer in the case of cis-isomers than transisomers. This indicates that cis-isomers of the cyclopentane $\beta$-amino acids studied $(1 R, 2 S$ and $1 S, 2 R)$ form kinetically more stable complexes with $\beta$-cyclodextrin than the trans-isomers.

\section{Differentiation of Enantiomeric \\ 2-Aminocyclohexanecarboxylic Acids \\ (Cyclohexane $\beta$-Amino Acids)}

The differentiation of the cyclohexane $\beta$-amino acids (5-8) was also successful. Although a great difference between the enantiomers was not achieved, the selectivities are moderate (Table 2). A greater selectivity was achieved for cis-isomers than for trans-isomers in the case of $(+)-$ and $(-)$-crown ether derivatives. The flexibility of the cyclohexane skeleton must have affected the results obtained, because in the case of cyclopentane $\beta$-amino acids (1-4), better enantioselectivity was obtained for the trans-isomers than for the cis-isomers. Again, when the (+)-crown ether derivative was used as a host compound, the selectivities were over one, indicating that complexes with $1 R, 2 S$ and $1 R, 2 R$ isomers change the guest faster than the corresponding complexes with $1 S, 2 R$ and $1 S, 2 S$ isomers. When the (-)crown ether derivative was used as a host compound, the selectivity values obtained were below one, as in the case of cyclopentane $\beta$-amino acids (Table 1).

When $\beta$-cyclodextrin was used as a host compound, the results differed again. ESI mass spectra of ion/

Table 2. Chiral selectivities $\left(\mathrm{S}_{\mathrm{c}}\right)$ for enantiomeric 2-aminocyclohexane carboxylic acids studied (5-8)

\begin{tabular}{lcc}
\hline \multirow{2}{*}{ Host } & \multicolumn{2}{c}{ Chiral selectivity $\left(\mathrm{S}_{\mathrm{c}}\right)^{\mathrm{a}}$} \\
\cline { 2 - 3 } & $\begin{array}{c}\text { cis-Isomers } \\
(1 R, 2 S) /(1 S, 2 R)\end{array}$ & $\begin{array}{c}\text { trans-Isomers } \\
(1 R, 2 R) /(1 S, 2 S)\end{array}$ \\
\hline \hline (+)-Crown derivative & $1.84 \pm 0.04$ & $1.25 \pm 0.03$ \\
(-)-Crown derivative & $0.50 \pm 0.01$ & $0.80 \pm 0.02$ \\
$\beta$-Cyclodextrin & Fast: $0.90 \pm 0.09$ & Fast: $2.01 \pm 0.28$ \\
& Slow: $0.71 \pm 0.05$ & Slow: $0.52 \pm 0.09$ \\
\hline
\end{tabular}

aSelectivity is obtained as ratio of rate constants $\left(k_{\mathrm{RS}} / k_{\mathrm{SR}}\right.$ and $\left.k_{\mathrm{RR}} / k_{\mathrm{SS}}\right)$.

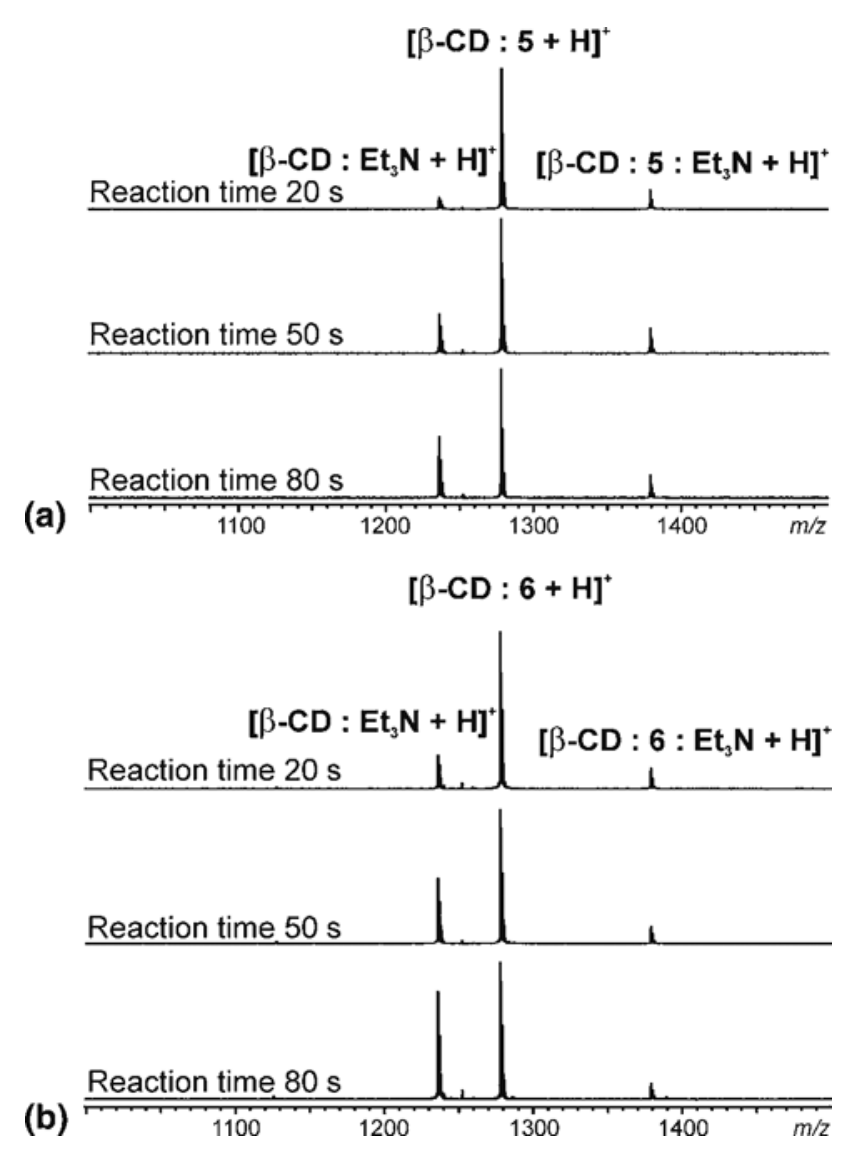

Figure 5. ESI mass spectra of ion/molecule reactions of hostguest complexes of $\beta$-cyclodextrin and (a) $1 R, 2 S$ - and (b) $1 S, 2 R$ cyclohexane $\beta$-amino acids (5 and 6) with triethylamine $\left(\mathrm{Et}_{3} \mathrm{~N}\right)$.

molecule reactions of host-guest complexes of $\beta$ cyclodextrin and $1 R, 2 S$ and $1 S, 2 R$ cyclohexane $\beta$-amino acids (5 and 6) with triethylamine $\left(\mathrm{Et}_{3} \mathrm{~N}\right)$ are presented in Figure 5. In this case, the abundance of the trimeric complex $\left([\text { Host: } \beta \text {-AC:Reagent }+\mathrm{H}]^{+}\right.$) ion was higher than in the case of the cyclopentane $\beta$-amino acids. This behavior might be due to the flexibility of cyclohexane $\beta$-amino acids compared with the more rigid cyclopentane $\beta$-amino acids. Because of this flexibility, the position of the cyclohexane $\beta$-amino acid enantiomer in the host-guest complex allows the addition of the neutral reagent, thus generating a stable trimeric complex ion [Host: $\beta$-AC:Reagent $+\mathrm{H}]^{+}$. In the case of longer reaction times, however, the abundance of the trimeric complexes was observed to decrease.

Corresponding reaction rate plots are presented in Figure 6 . The reaction plots of the enantiomeric $\beta$-amino acids show bimodal behavior showing the existence of fast $\left(k_{\text {fast }}\right)$ and slow $\left(k_{\text {slow }}\right)$ reacting complexes. The reason for this kind of behavior might be due to possibility that more than one ion population is present and/or there are some "hot" segments in ion population, which are reacting quickly away [34, 42]. This same kind of behavior was also observed in our earlier studies of differentiation of diastereomeric $\beta$-amino acids, for example cyclopentane and cyclohexane 

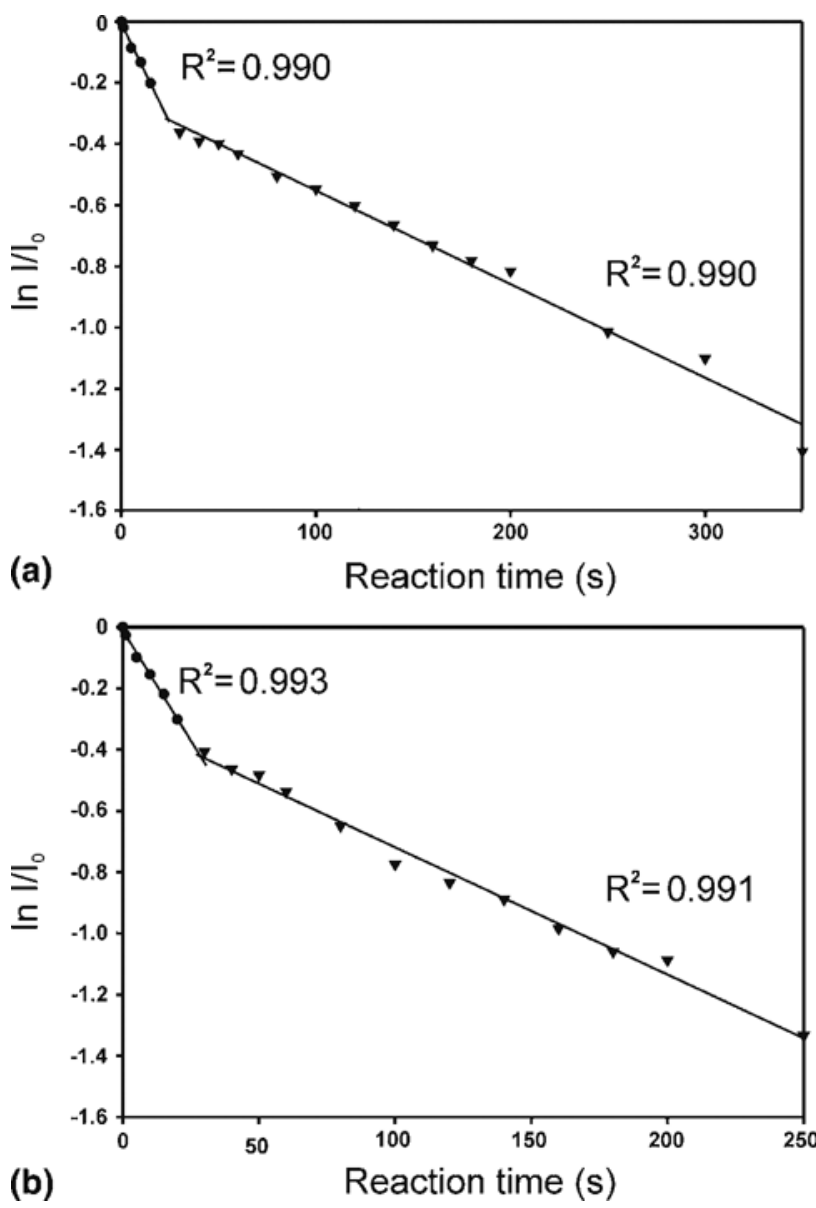

Figure 6. Rate plots for reactions between complexes of $\beta$ cyclodextrin with (a) $1 R, 2 S$ - and (b) $1 S, 2 R$-cyclohexane $\beta$-amino acids (5 and 6 ) and triethylamine.

$\beta$-amino acids, when host-guest complexes and ion/ molecule reactions were utilized [38, 43]. Moreover, Lebrilla et al. have observed this same kind of behavior, especially when studying some trimeric complexes [40, 41]. It is assumed that with short reaction times, trimeric complexes are kinetically favored. In the case of longer reaction times, the reaction pathway forming the new host-guest complex persists after zwitterion formation has diminished [41]. Because of this kind of behavior, the enantioselectivities are calculated based on both fast and slow reaction complexes. However, since the total amount of the fast-reacting complexes was small, the emphasis is on the slower reacting complexes.

Overall, a satisfactory differentiation was achieved for the enantiomeric cyclohexane $\beta$-amino acids studied (especially for cis-isomers) using (+)- and (-)-crown ether derivatives as host compounds. Interestingly, when $\beta$-cyclodextrin was used as the host compound, higher enantioselectivity was achieved for the transisomers. Although it has been a challenging task to differentiate flexible cyclohexane $\beta$-amino acid isomers, this study has shown that it is possible to achieve enantioselectivity by using different chiral host compounds in ion/molecule reactions.

\section{Conclusions}

The chiral differentiation of enantiomers including two chiral centers is successfully performed in this study using host-guest complexes and ion/molecule reactions. Four enantiomeric pairs of $\beta$-amino acids were studied; cis$(1 R, 2 S)-$, cis-(1S,2R)-, trans-(1R,2R)-, and trans-(1S,2S)-2aminocyclopentanecarboxylic acids (cyclopentane $\beta$-amino acids), and cis-(1R,2S)-, cis-(1S,2R)-, trans-(1R,2R)-, and trans-(1S,2S)-2-aminocyclohexanecarboxylic acids (cyclohexane $\beta$-amino acids). The degree of enantioselectivity $\left(\mathrm{S}_{\mathrm{c}}\right)$ was evaluated by using three different kinds of host compounds: (+)-(18-Crown-6)-2,3,11,12-tetracarboxylic acid, (-)-(18-Crown-6)-2,3,11,12-tetracarboxylic acid, and $\beta$ cyclodextrin.

The results obtained showed that chiral differentiation of the $\beta$-amino acids studied was achieved by using all three host compounds. For cyclopentane $\beta$-amino acids (1-4), the highest enantioselectivity was achieved with (+)- and (-)-crown ether derivatives as host compounds. When $\beta$-cyclodextrin was used as the host compound, an additional trimeric complex of ions [Host: $\beta$-AC:Reagent $+\mathrm{H}]^{+}$was observed in mass spectra. The abundance of these trimeric complexes, however, did not contribute to the reaction rate plots obtained. In addition, better differentiation of enantiomers was obtained for trans-isomers $(1 R, 2 R$ and $1 S, 2 S)$ than for cis-isomers $(1 R, 2 S$ and $1 S, 2 R)$.

Enantiomeric differentiation of cyclohexane $\beta$-amino acids (5-8) was also successful. When the (+)-crown ether derivative was used as the host compound, the enantioselectivities obtained were over one. In the case of the (-)-crown ether derivative, the enantioselectivities obtained were below one. This is a very logical behavior, showing the validity of the results, because the only difference was the optically reversed host compound. The same kind of behavior was also observed in the case of cyclopentane $\beta$-amino acids studied.

When $\beta$-cyclodextrin was used as a host compound, the abundance of the trimeric complexes with cyclohexane $\beta$-amino acids was much higher than in the case of the cyclopentane $\beta$-amino acids used in this study. This derives from the flexibility of the cyclohexane compared with the more rigid structure of the cyclopentane skeleton. The existence of the trimeric complexes contributed to the reaction rate plots, thus forming two linear regions with two different slopes (fast and slow reacting complexes). However, the amount of the fast reacting complexes was small; therefore the emphasis is on the slower reacting complexes.

\section{Acknowledgments}

The authors gratefully acknowledge funding provided by the Graduate School of Organic Chemistry and Chemical Biology, Finland. 


\section{References}

1. Steed, J. W.; Atwood, J. L. Supramolecular Chemistry; Wiley: Chichester, England, 2000; p. 88-92, 321-334.

2. Vögtle, F. Supramolecular Chemistry, Wiley: Chichester, England, 1991; p. 27-32.

3. Li, S.; Purdy, W. C. Cyclodextrins and their applications in analytical chemistry. Chem. Rev. 1992, 92, 1457-1470.

4. Connors, K. A. The stability of cyclodextrin complexes in solution. Chem. Rev. 1997, 97, 1325-1357.

5. Sallas, F.; Darcy, R. Amphiphilic cyclodextrins - Advances in synthesis and supramolecular chemistry. Eur. J. Org. Chem. 2008, 957-969.

6. Ward, T. J. Chiral separations. Anal. Chem. 2002, 74, 2863-2872.

7. Hembury, G. A.; Borovkov, V. V.; Inoue, Y. Chirality-sensing supramolecular systems. Chem. Rev. 2008, 108, 1-73.

8. Hoffmann, C. V.; Pell, R.; Lämmerhofer, M.; Lindner, W. Synergistic effects on enantioselectivity of zwitterionic chiral stationary phases for separations of chiral acids, bases, and amino acids by HPLC. Anal. Chem. 2008, 80, 8780-8789.

9. Péter, A.; Fülöp, F. High-performance liquid chromatographic method for the separation of isomers of cis- and trans-2-amino-cyclopentane-1carboxylic acid. J. Chromatogr. A 1995, 715, 219-226.

10. Nishi, H.; Nakamura, K.; Nakai, H.; Sato, T. Separation of enantiomers and isomers of amino compounds by capillary electrophoresis and high-performance liquid chromatography utilizing crown ethers. J. Chromatogr. A 1997, 757, 225-235.

11. Hyun, M. H.; Tan, G.; Xue, J. Y. Unusual resolution of N-(3,5dinitrobenzoyl)- $\alpha$-amino acids on a chiral stationary phase based on (+)-(18-Crown-6)-2,3,11,12-tetracarboxylic acid. J. Chromatogr. A 2005, 1097, 188-191.

12. Berkecz, R.; Ilisz, I.; Fülöp, F.; Pataj, Z.; Hyun, M. H.; Péter, A. High-performance chromatographic enantioseparation of $\beta$-3homo-amino acid stereoisomers on a (+)-(18-crown-6)-2,3,11,12tetracarboxylic acid-based stationary phase. J. Chromatogr. A 2008, 1189, 285-291.

13. Tanaka, Y.; Otsuka, K.; Terabe, S. Separation of enantiomers by capillary electrophoresis-mass spectrometry employing a partial filling technique with a chiral crown ether. J. Chromatogr. A 2000, 875, 323-330.

14. Zhou, L.; Lin, Z.; Reamer, R. A.; Mao, B.; Ge, Z. Stereoisomeric separation of pharmaceutical compounds using CE with a chiral crown ether. Electrophoresis 2007, 28, 2658-2666.

15. Bunke, A.; Jira, T. Use of cationic cyclodextrin for enantioseparation by capillary electrophoresis. J. Chromatogr. A 1998, 798, 275-280.

16. Szejtli, J.; Juvancz, Z. The role of cyclodextrins in chiral selective chromatography. Trends Anal. Chem. 2002, 21, 379-388.

17. Sicoli, G.; Jiang, Z.; Jicsinsky, L.; Schurig, V. Modified linear dextrins ("acyclodextrins") as new chiral selectors for the gas-chromatographic separation of enantiomers. Angew. Chem. Int. Ed. 2005, 44, 4092-4095.

18. Patrick, J. S.; Kotiaho, T.; McLuckey, S. A.; Cooks, R. G. Thermochemical determinations by the kinetic method. Mass Spectrom. Rev. 1994, 13, 287-339.

19. Tao, W. A.; Zhang, D.; Wang, F.; Thomas, P. D.; Cooks, R. G. Kinetic resolution of D,L-amino acids based on gas-phase dissociation of Copper(II) complexes. Anal. Chem. 1999, 71, 4427-4429.

20. Speranza, M. Enantioselectivity in gas-phase ion-molecule reactions. Int. J. Mass Spectrom. 2004, 232, 277-317.

21. Vincenti, M. Host-guest chemistry in the mass spectrometer. J. Mass Spectrom. 1995, 30, 925-939.

22. Schalley, C. A. Molecular recognition and supramolecular chemistry in the gas phase. Mass Spectrom. Rev. 2001, 20, 253-309.

23. Brodbelt, J. S. Analytical applications of ion-molecule reactions. Mass Spectrom. Rev. 1997, 16, 91-110.

24. Schug, K. A.; Lindner, W. Chiral molecular recognition for the detection and analysis of enantiomers by mass spectrometric methods. J. Sep. Sci. 2005, 28, 1932-1955.
25. Sawada, M. Chiral recognition detected by fast atom bombardment mass spectrometry. Mass Spectrom. Rev. 1997, 16, 73-90.

26. Takai, Y.; Yamada, H.; Nishida, J.; Kaneda, T.; Arakawa, R.; Okamoto M.; Hirose, K.; Tanaka, T.; Naemura, K.; Sawada, M. Chiral amino acid recognition detected by electrospray ionization (ESI) and fast atom bombardment (FAB) mass spectrometry (MS) coupled with the enantiomer-labeled (EL) guest method. J. Chem. Soc. Perkin Trans 21998 701-710.

27. Daniel, J. M.; Friess, S. D.; Rajagopalan, S.; Wendt, S.; Zenobi, R. Quantitative determination of noncovalent binding interactions using soft ionization mass spectrometry. Int. J. Mass Spectrom. 2002, 216, 1-27.

28. Kellersberger, K. A.; Dejsupa, C.; Liang, Y.; Pope, R. M.; Dearden, D. V. Gas phase studies of ammonium-cyclodextrin compounds using Fourier transform ion cyclotron resonance. Int. J. Mass Spectrom. 1999, 193 181-195.

29. Chu, I. H.; Bradshaw, J. S.; Huszthy, P.; Izatt, R. M.; Dearden, D. V. Chiral host-guest recognition in an ion-molecule reaction. J. Am. Chem. Soc. 1993, 115, 4318-4320.

30. Dejsupa, C.; Liang, Y.; Bradshaw, J. S.; Izatt, R. M.; Dearden, D. V. Intrinsic contributions to chiral recognition: discrimination between enantiomeric amines by dimethyldiketopyridino-18-crown-6 in the gas phase. J. Am. Chem. Soc. 1997, 119, 353-359.

31. Liang, Y.; Bradshaw, J. S.; Dearden, D. V. The thermodynamic basis for enantiodiscrimination: Gas-phase measurement of the enthalpy and entropy of chiral amine recognition by dimethyldiketopyridino-18crown-6. J. Phys. Chem. A 2002, 106, 9665-9671.

32. Ramirez, J.; Ahn, S.; Grigorean, G.; Lebrilla, C. B. Evidence for the formation of gas-phase inclusion complexes with cyclodextrins and amino acids. J. Am. Chem. Soc. 2000, 122, 6884-6890.

33. Ahn, S.; Ramirez, J.; Grigorean, G.; Lebrilla, C. B. Chiral recognition in gas-phase cyclodextrin: amino acid complexes-is the three point interaction still valid in the gas phase? J. Am. Soc. Mass Spectrom. 2001, $12,278-287$.

34. Grigorean, G.; Lebrilla, C. B. Enantiomeric analysis of pharmaceutical compounds by ion/molecule reactions. Anal. Chem. 2001, 73, 1684-1691.

35. Grigorean, G.; Cong, X.; Lebrilla, C. B. Chiral analysis of peptides by ion/molecule reactions. Int. J. Mass Spectrom. 2004, 234, 71-77.

36. Fülöp, F. The chemistry of 2-aminocycloalkanecarboxylic acids. Chem. Rev. 2001, 101, 2181-2204.

37. Fülöp, F.; Martinek, T. A.; Tóth, G. K. Application of alicyclic $\beta$-amino acids in peptide chemistry. Chem. Soc. Rev. 2006, 35, 323-334.

38. Hyyryläinen, A. R. M.; Pakarinen, J. M. H.; Fülöp, F.; Vainiotalo, P. Differentiation of diastereomeric cyclic $\beta$-amino acids by varying the neutral reagent in ion/molecule reactions studied by electrospray ionization Fourier transform ion cyclotron resonance mass spectrometry. Rapid Commun. Mass Spectrom. 2008, 22, 1-8.

39. de Koning, L. J.; Nibbering, N. M. M.; van Orden, S. L.; Laukien, F. H. Mass selection of ions in a Fourier transform ion cyclotron resonance trap using correlated harmonic excitation fields (CHEF). Int. J. Mass Spectrom. 1997, 165/166, 209-219.

40. Ahn, S.; Cong, X.; Gronert, S.; Lebrilla, C. B. Zwitterion formation in gas-phase cyclodextrin complexes. J. Am. Soc. Mass Spectrom. 2005, 16 , 166-175.

41. Cong, X.; Czerwieniec, G.; McJimpsey, E.; Ahn, S.; Troy, F. A.; Lebrilla C. B. Structural relationships in small molecule interactions governing gas-phase enantioselectivity and zwitterionic formation. J. Am. Soc. Mass Spectrom. 2006, 17, 442-452.

42. Botta, B.; Subissati, D.; Tafi, A.; Monache, G. D.; Filippi, A.; Speranza, M. Cavity effects on the enantioselectivity of chiral amino[4]resorcinarene stereoisomers. Angew. Chem. Int. Ed. 2004, 43, 4767-4770.

43. Hyyryläinen, A. R. M.; Pakarinen, J. M. H.; Stájer, G.; Fülöp, F.; Vainiotalo, P. Diastereochemical differentiation of $\beta$-amino acids using host-guest complexes studied by Fourier transform ion cyclotron resonance mass spectrometry. J. Am. Soc. Mass Spectrom. 2007, 18, $1038-1045$. 